\title{
Spatio-temporal distribution characteristics of cysticercosis from 2000 to 2014 in Dali, Yunnan Province, China
}

\author{
Huanzhang Li, ${ }^{1,2}$ Xinzhong Zang, ${ }^{1}$ Xiaokang Hu, ${ }^{1}$ Eniola Michael Abe, ${ }^{1}$ Menbao Qian, \\ Jingbo Xue, ${ }^{1}$ Yingdan Chen, ${ }^{1}$ Changhai Zhou, ${ }^{1}$ Yuhua Liu, ${ }^{3}$ Shizhu Li ${ }^{1}$ \\ ${ }^{I}$ National Institute of Parasitic Diseases, Chinese Center for Disease Control and Prevention, Key \\ Laboratory on Biology of Parasite and Vector, Ministry of Health, National Center for International \\ Research on Tropical Diseases, WHO Collaborating Center for Tropical Diseases, Shanghai; \\ ${ }^{2}$ Dandong health service center, Liaoning Province; ${ }^{3}$ Dali Prefectural Institute of Research and Control \\ on Schistosomiasis, Yunnan Province, China
}

\begin{abstract}
Cysticercosis remains a public health problem in China, with disease prevalence attributed to poor socio-economic and public health conditions. This parasitic food-borne disease was prioritized for effective control following implementation of the national surveys on parasitic diseases carried out in China. We predicted the cysticercosis distribution in Dali, Yunnan Province by assessing spatio-temporal distribution characteristics between 2000 and 2014 to better understand the trend of the disease incidence. A database of cysticercosis cases was provided by the clinical department at the Dali Prefectural Institute of Research and Control of Schistosomiasis. Describing the epidemiological features of cysticercosis and analyzing its spatiotemporal distribution of cases using mapping, scanning and spatial autocorrelation analysis, our findings found a total of 3,347 patients with cysticercosis infection, neurocysticercosis in particular. Cysticercosis prevalence was the highest among young and middle-aged male farmers, and also predominant among the
\end{abstract}

Correspondence: Shizhu Li, National Institute of Parasitic Diseases, Chinese Center for Disease Control and Prevention, Shanghai 200025, China.

E-mail: Lisz@chinacdc.cn

Key words: Cysticercosis; spatio-temporal; distribution; characteristics; Yunnan Province; China.

Conflict of interests: The authors declare no potential conflict of interests.

Received for publication: 29 September 2019.

Accepted for publication: 24 February 2020.

(C) Copyright: the Author(s), 2020

Licensee PAGEPress, Italy

Geospatial Health 2020; 15:815

doi:10.4081/gh.2020.815

This article is distributed under the terms of the Creative Commons Attribution Noncommercial License (CC BY-NC 4.0) which permits any noncommercial use, distribution, and reproduction in any medium, provided the original author(s) and source are credited.
Bai nationality. Three aggregation areas were identified during the period 2000-2014. Hotspot analysis implicated Dali City, Eryuan County and Yangbi County between 2000 and 2007, with areas gradually shifting towards the western and northern parts of the province. The hotspot map indicated that Eryuan County was a constant problem with respect to cysticercosis. The results indicated three cysticercosis clusters in Dali that could be attributed to environmental factors and unhealthy lifestyles. Multi-sectoral control initiatives are, therefore, recommended in these areas to effectively control and prevent cysticercosis among the population.

\section{Introduction}

Cysticercosis is a parasitic infection caused by Taenia solium larvae. Humans play the role of definitive host, but can also be the intermediate host. In the latter case, infection in human occurs when the parasite eggs are ingested via the faecal-oral route, especially by ingesting contaminated food and water (Garcia et al., 2003). The T. solium larvae can parasitize a variety of human tissues and organs, commonly the central nervous system, causing serious health challenges including seizures (Garcia and Del Brutto, 2003; Mahanty and Garcia, 2010; Raina et al., 2012; Wu et al., 2013), intracranial hypertension and hypophrenia when not treated. Cysticercosis is one of the Neglected Tropical Diseases (NTDs), associated with poor socio-economic status and public health conditions and listed as an important global food-borne parasitic disease by the United Nation's Food and Agriculture Organization (FAO) and World Health Organization (WHO), (Sheng \& Yang, 2011; Trevisan et al., 2017).

The disease is widespread and it remains a global public health concern in many developing countries in Latin America, sub-Saharan Africa and Asia (Flisser and Correa, 2010). Neurocysticercosis (NCC is the most common outcome of cysticercosis infection and the main cause of secondary epilepsy (Tang et al., 2012; Xu, 2016; Gripper and Welburn, 2017). Cysticercosis was once a disease with serious public health concern in People's Republic of China, but its endemicity has been significantly controlled with the implementation of the national parasitic diseases control programme. However, disease prevalence is still very high in western China despite control efforts. Yunnan is one of the areas implicated with high cysticercosis 
incidence in China with prominent widespread infection.

Cysticercosis was prioritized for effective prevention in the country, following the first nationwide investigation of human parasites infection in China. Taeniasis prevalence in Dali, Yunnan Province decreased from $6.9 \%$ over $4.2 \%$ to $0.9 \%$ in 1987,1988 and 1992, respectively. However, the prevalence in individual villages could be as high as $28.3 \%$ (Gong et al., 1997). Another study conducted a three-month survey of 436 people with parasitic infections in Pumi inhabited areas of Yunnan. Findings indicated that $35.9 \%$ of sampled faeces contained T. solium eggs (Zhang et al., 1991). Investigation of 3918 people using ELISA in six counties of southern and western Yunnan between 1992 and 2000 revealed that 154 were positive for cysticercosis antibody with $3.93 \%$ seroprevalence rate (Tang et al., 2012).

Results obtained from the National Survey on Distribution of Human Parasites in China between 1988 and 1992 show that cysticercosis was distributed in 27 provinces/autonomous regions/municipalities across the country (Yu et al., 1994). In addition, in the period 2001-2004, the Coordinating Office of the National Survey on the Important Parasitic Diseases in China (2005) found a higher rate of anti-cysticercosis antibody than the national average $(0.6 \%)$ in 10 provinces/autonomous regions/municipalities, among them Shanxi Province with $3.4 \%$ and Fujian Province with $2.0 \%$. However, the disease has been effectively controlled nationwide following continuous improvement in the country's development in the last decade. The prevalence is, however, still very high in western China, particularly in the provinces of Yunnan and Sichuan.

It was therefore decided to carry out an assessment of the cysticercosis distribution by spatio-temporal analysis in Dali, Yunnan Province based on database information of cysticercosis registered patients in Dali Prefecture from 2000 to 2014.

\section{Materials and methods}

\section{Study area}

The work was carried out in Dali Prefecture, which is located in the western part of Yunnan Province with jurisdiction over Dali City and 11 counties: Yangbi, Xiangyun, Binchuan, Midu, Weishan, Nanjiang, Yongping, Yunlong, Eryuan, Jianchuan and Heqing. Dali Prefecture comprises also a large number of townships, including 11 ethnic ones (Zhang, 2017).

\section{Data Collection}

Case investigation, data collection and sorting of information were carried out between March 2017 and March 2018 at the clinical department of Dali Prefectural Institute of Research and Control on Schistosomiasis, Yunnan Province. The Institute serves as reference center for the treatment of patients with parasitic diseases (including cysticercosis) in Dali and surrounding areas (Zang et al., 2019).

Cysticercosis cases considered in this study were selected according to the diagnostic criteria given in "Diagnosis of cysticercosis (standard number: WS381-2012)" issued by the Ministry of Health for data collected between 2010 and 2014. In addition to detailed long-term epidemiological data including patient name, gender, age, and time of registration and address, information on symptoms, radiographic and serological exami- nation results were accessed. Preliminary screening was conducted according to initial data based on available epidemiological information and diagnosis were recorded for subsequent analysis because patients with cysticercosis need to undergo a standardized treatment schedule with several courses of clinical treatment.

According to the statistics code of the National Bureau of Statistics, People's Republic of China (2016), the names of the counties in Dali Prefecture were extracted as the associated fields, digital maps were matched. A geographic information database of cysticercosis in Dali Prefecture was established, including 15 years' information which describes yearly distribution and hospitalization of cysticercosis patients.

\section{Spatio-temporal cluster analysis}

In this study, Moran's I (Mitchell, 2005) was used to evaluate the global spatial autocorrelation of cysticercosis in Dali Prefecture. The ArcGIS, v. 10.3 software (ESRI, Redlands, CA, USA) was used to compute and analyze the space-time pattern of the disease. The input feature was the number of hospitalizations per 100,000 population, while the output features categories included Moran's $I, z$-score, $p$-value, and cluster/outlier (CO type). Geographic coordinates (latitude and longitude) of the cases' address were extracted and SaTScan, v9.6 (Kulldorff, 2018) spatial scanning method was used to identify the spatial aggregation of cysticercosis taking the number of people infected per year into account. We processed data in Microsoft Excel 2013 in order to analyze the endemic status distribution and epidemiological features of cysticercosis cases over the 15 years. Then, these data were imported into ArcGIS and SaTScan to perform both spatial and temporal analysis. Official shapefiles (.shp) for Dali Prefecture were obtained from the National Institute of Parasitic Diseases (NIPD) and Chinese Center for Disease Control and prevention (China CDC).

\section{Results}

\section{Endemic status}

Between 2000 and 2014, a total of 3,347 new cases were diagnosed and registered in Dali Prefectural Institute of Research and Control on Schistosomiasis as cysticercosis cases. The highest number of cases was recorded in 2008 with 282 cases and the lowest in 2014 with 156 cases. Figure 1 shows the numbers of registered cases between 2000 and 2014. These numbers were generally $>200$ most of the time but there was an annual decline in the numbers after 2012. Among these cases, there were 3,201 with NCC (95.6\%, 3201/3347), 127 mixed type cysticercosis $(3.8 \%, 127 / 3347), 17$ with cutaneous cysticercosis $(0.5 \%$, $17 / 3347)$ and two cases of tongue cysticercosis $(0.1 \%, 2 / 3347)$.

\section{Epidemiological features}

The number of reported diagnostic cases showed that $56.4 \%$ (1887/3347) were male, there were more male patients than females each year, and the most affected age group was the one comprising the 0-14 years olds, which accounted for $27.2 \%$ (Table 1). The Bai nationality accounted for 56.8\% (1900/3347) of all cysticercosis data obtained and farmers were the most susceptible $(57.0 \% ; 1908 / 3347)$, (Figures 2 and 3). The number of 
Table 1. Demographic features of cysticercosis cases reported 2000-2014.

\begin{tabular}{|c|c|c|c|c|c|c|c|c|c|c|c|c|c|c|c|c|}
\hline Feature & 2000 & 2001 & 2002 & 2003 & 2004 & 2005 & 2006 & 2007 & 2008 & 2009 & 2010 & 2011 & 2012 & 2013 & 2014 & Total \\
\hline \multicolumn{17}{|c|}{ By gender } \\
\hline Male & 109 & 141 & 126 & 107 & 113 & 142 & 141 & 129 & 155 & 156 & 125 & 137 & 99 & 111 & 96 & 1,887 \\
\hline Female & 96 & 102 & 87 & 82 & 100 & 111 & 113 & 102 & 127 & 118 & 101 & 115 & 95 & 51 & 60 & 1,460 \\
\hline \multicolumn{17}{|c|}{ By age group } \\
\hline $0-14$ & 68 & 94 & 83 & 62 & 76 & 87 & 86 & 67 & 60 & 49 & 44 & 57 & 23 & 26 & 28 & 910 \\
\hline $15-29$ & 59 & 70 & 64 & 56 & 44 & 69 & 54 & 75 & 75 & 72 & 78 & 56 & 55 & 28 & 33 & 888 \\
\hline $30-44$ & 49 & 47 & 38 & 48 & 49 & 66 & 67 & 51 & 93 & 96 & 60 & 80 & 59 & 47 & 40 & 890 \\
\hline $45-59$ & 26 & 29 & 26 & 24 & 25 & 20 & 37 & 31 & 42 & 45 & 36 & 49 & 43 & 46 & 35 & 514 \\
\hline$>60$ & 4 & 3 & 2 & 6 & 16 & 11 & 10 & 7 & 10 & 9 & 8 & 10 & 14 & 15 & 20 & 145 \\
\hline
\end{tabular}

farmer patients peaked in 2009 and declined thereafter, only amounting to 100 cases in 2014. Student patients accounted for $28.3 \%(946 / 3347)$, with the number of patients in this group decreasing year-by-year since 2001 reaching the lowest value (30 cases) in 2013. There were $193(5.8 \%)$ preschool children and $136(4.1 \%)$ officials.

\section{Spatio-temporal distributions}

Figure 4 shows the distribution trend of cysticercosis cases per 100,000 people in different townships in Dali from 2000 to 2014. Overall, the number of cases showed a decreasing trend over the years. The number of hospitalized patients in Fengyi Town was the highest of the townships with $11.7 \%(390 / 3347)$ during the 15 years considered, while the highest number of hospitalized cases per 100,000 population was 8.70 in 2006 . Applying Moran's $I$, we found a state-wide, spatial autocorrelation of cysticercosis in Dali Prefecture over the whole study period and an indication of annual spatial aggregation. The probability of random occurrence was less than 0.01 and the distribution presented an aggregation phenomenon (Table 2). Space-time cluster analysis showed that the towns Fengyi and Xiaguan in the centre of the main spatial cysticercosis cluster (Table 3, Figure 5 ). The total number cases in Dali during the 7-year period from 2000 to 2006 was 1,570 , out of which 404 (25.7\%) were hospitalized. Overall, the number of hospitalizations per 100,000 population showed a general, downward trend over the period. The total number of hospitalizations between 2005 and 2011 was 490, and the number of hospitalizations in Yousuo Town was 124 $(25.3 \%)$. The total number of hospitalizations in the towns and the cluster area was $63(14.1 \%)$, i.e. 63 of the total hospitalizations (447) in the district between 2002 and 2008.

\section{Hotspot analysis}

The hotspots were mainly concentrated mostly in Dali's towns in Eryuan and YangBi counties, then gradually extending in to Jianchuan County including Shaxi, Diannan, Jinhua and other regions as well as towns in Heqing County, such as Songgui and Xiyi between 2000 and 2007. Moving westward to the townships Guanping, Baishi, Jiancao and Changxin in Yunlong County, the hotspot map shows that Eryuan County is always within the hotspot area. It should be noted that all the above-mentioned towns have geographical borders with Eryuan County (Figure 6).

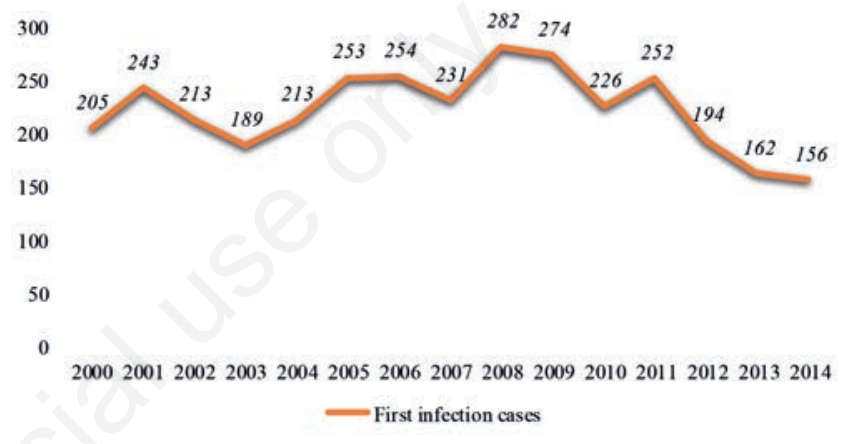

Figure 1. Reported cysticercosis cases from 2000 to 2014.

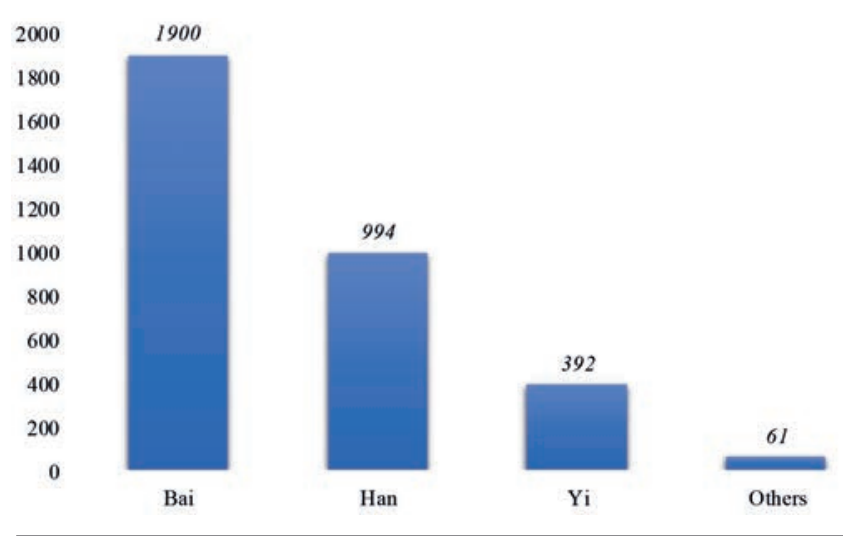

Figure 2. Case distribution by ethnicity.

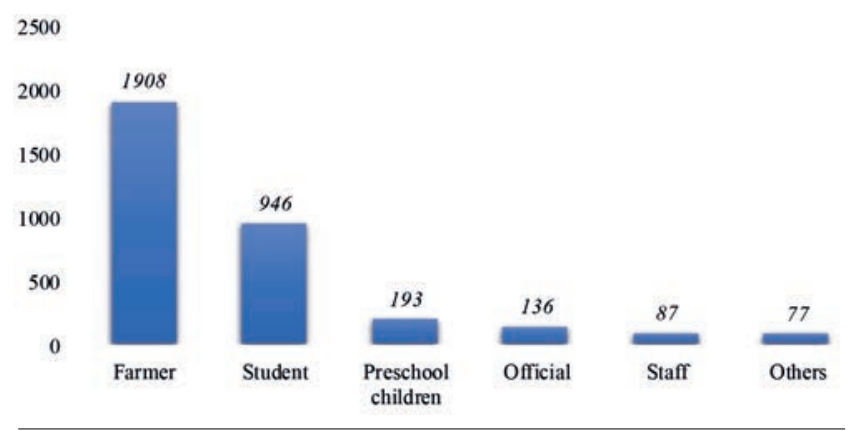

Figure 3. Case distribution by occupation. 


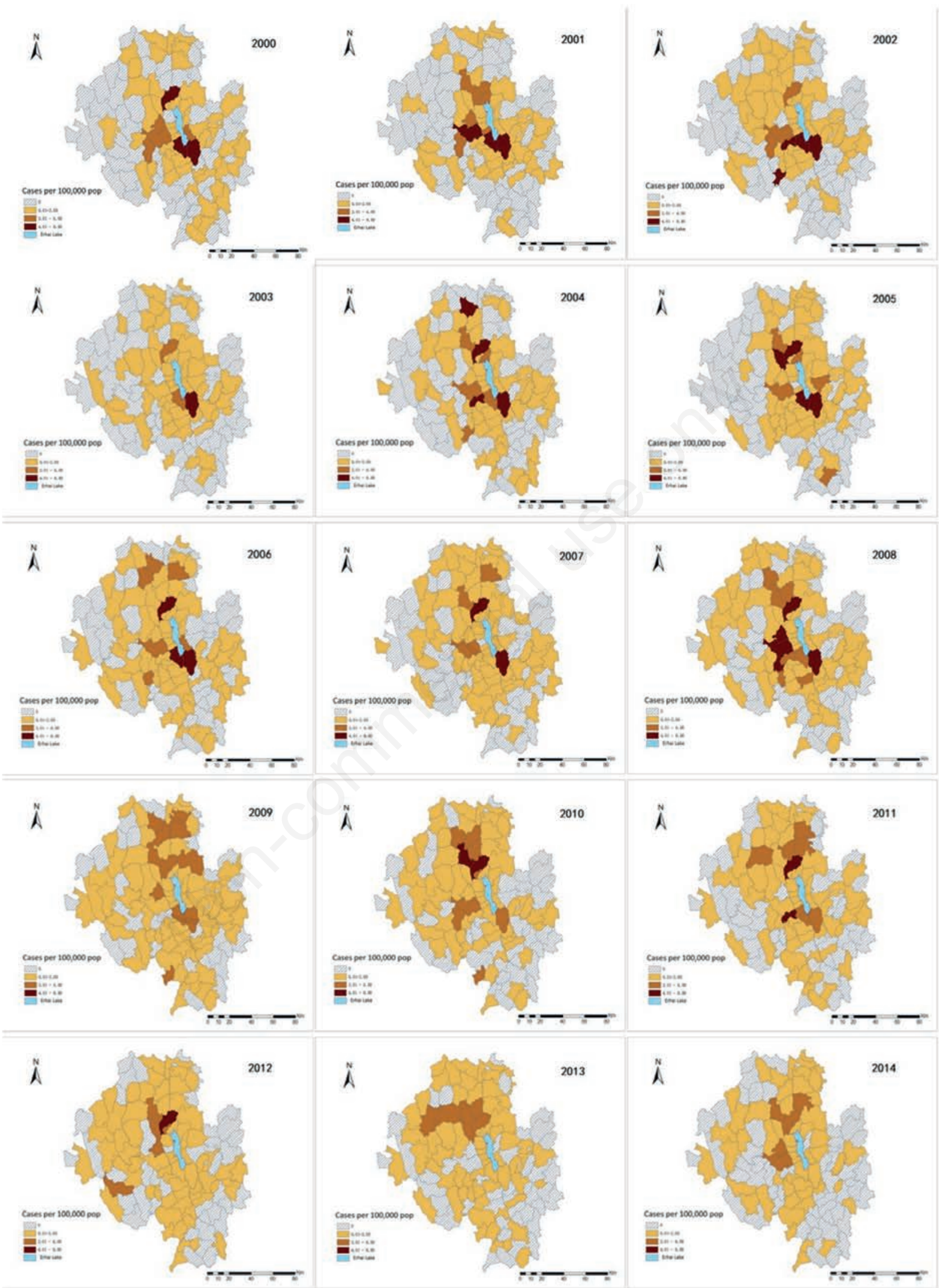

Figure 4. Number of hospitalized cases per 100,000 population in Dali, Yunnan Province in the period 2000-2014. 


\section{Discussion}

Analysis of the distribution characteristics of patients with cysticercosis hospitalized at township level in Dali Prefecture indicated that there were more cases in Dali City and the counties of Eryuan and Yangbi. The towns Xiaguan, Fengyi and Yousuo were implicated with high cysticercosis incidences. This indicates that there are challenges with control effectiveness in some rural areas which might be attributed to poor environmental sanitation, poor breeding of pigs and slaughter management, incomplete sewage treatment facilities, low utilization rate of toilets in the rural areas which makes cysticercosis a public health problem in our study area.

The Bai people accounts for about one-third of Dali population, which makes them the major tribe. People of Bai origin accounted for $56.8 \%$ of the patient population, which can be attributed to their unique custom of eating raw pork. Similar findings were earlier reported (Chen et al., 2007; Fang et al., 2009; Yu et al., 2015). Ensuring strict food safety system and animal quarantine can reduce infection greatly, but it cannot guarantee absolute safety of the food, so residents living in cysticercosis endemic areas need to be vigilant at all times.

Farmers, also, account for the highest proportion of infection. They are more likely to be exposed to infection risk factors from raw vegetables, contaminated water and the poor environmental

Table 2. Result of the correlation calculations.

\begin{tabular}{lccc}
\hline Year & Moran's I & Z-score & p-value \\
2000 & 0.19 & 3.24 & $<0.01$ \\
2001 & 0.39 & 6.28 & $<0.01$ \\
\hline 2002 & 0.28 & 4.76 & $<0.01$ \\
2003 & 0.21 & 3.69 & $<0.01$ \\
\hline 2004 & 0.27 & 4.34 & $<0.01$ \\
2005 & 0.29 & 4.65 & $<0.01$ \\
\hline 2006 & 0.23 & 3.98 & $<0.01$ \\
2007 & 0.24 & 4.08 & $<0.01$ \\
\hline 2008 & 0.20 & 3.19 & $<0.01$ \\
2009 & 0.23 & 3.62 & $<0.01$ \\
\hline 2010 & 0.30 & 4.78 & $<0.01$ \\
2011 & 0.25 & 4.17 & $<0.01$ \\
\hline 2012 & 0.32 & 5.04 & $<0.01$ \\
2013 & 0.34 & 5.32 & $<0.01$ \\
\hline 2014 & 0.26 & 4.16 & $<0.01$ \\
\hline
\end{tabular}

sanitation and living conditions in the rural areas. The high reproductive capacity of the tapeworm and the ability of the parasite's eggs to adapt and survive different environmental conditions make people in areas with poor sanitation and hygiene more susceptible to cysticercosis infection (Zammarchi et al., 2013).

Our findings using spatial and temporal distribution analysis indicate ten towns where cysticercosis is concentrated in Dali Prefecture, in particular Dali City, Eryuan County and Heqing County. Cysticercosis epidemics in these areas are influenced by a combination of environmental factors, such as poor living conditions and the fact that the majority of the local residents are farmers who are at a high risk of contact with parasite eggs. It is, therefore, imperative that authorities develop a stratagem applying control measures to key populations and areas prone to cluster development, conduct active case screening aimed at achieving early detection of cysticercosis cases through early diagnosis and early treatment.

T. solium is transmission are closely related to human behavioural factors. There is need to establish disease control and surveillance units at the local level to persistently create awareness using health education through different communication platforms such as distribution of leaflets, use of bill boards in strategic locations, media outlets and outreach. This would help people to switch to a better hygiene culture and healthy lifestyle, thereby reducing transmission and the risk of infection.

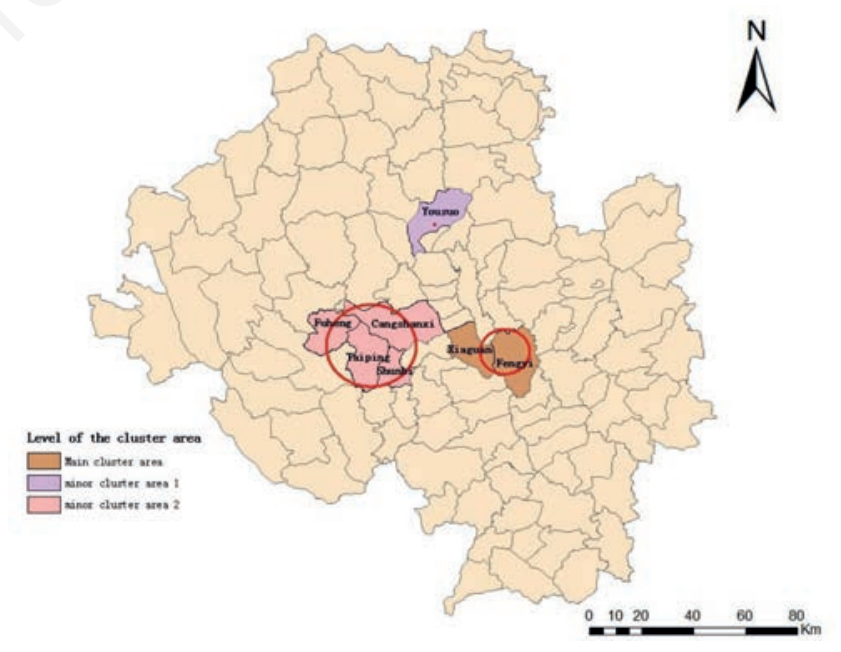

Figure 5. Spatio-temporal changes of cysticercosis distribution over the period 2000-2014.

Table 3. Spatio-temporal clusters discovered.

\begin{tabular}{|c|c|c|c|c|c|c|c|}
\hline Cluster type & Cluster time & Centre & Radius (km) & Num-ber & Relative risk & P-value & LLR* $^{*}$ \\
\hline Main & $2000-2006$ & Fengyi & 8.64 & 2 & 5.34 & $<0.01$ & 331.17 \\
\hline Minor 1 & $2005-2011$ & Yousuo & 0 & 1 & 5.78 & $<0.01$ & 116.53 \\
\hline Minor 2 & $2002-2008$ & Taiping & 15.63 & 4 & 2.24 & $<0.01$ & 16.06 \\
\hline
\end{tabular}

*Log-likelihood ratio, LLR. 

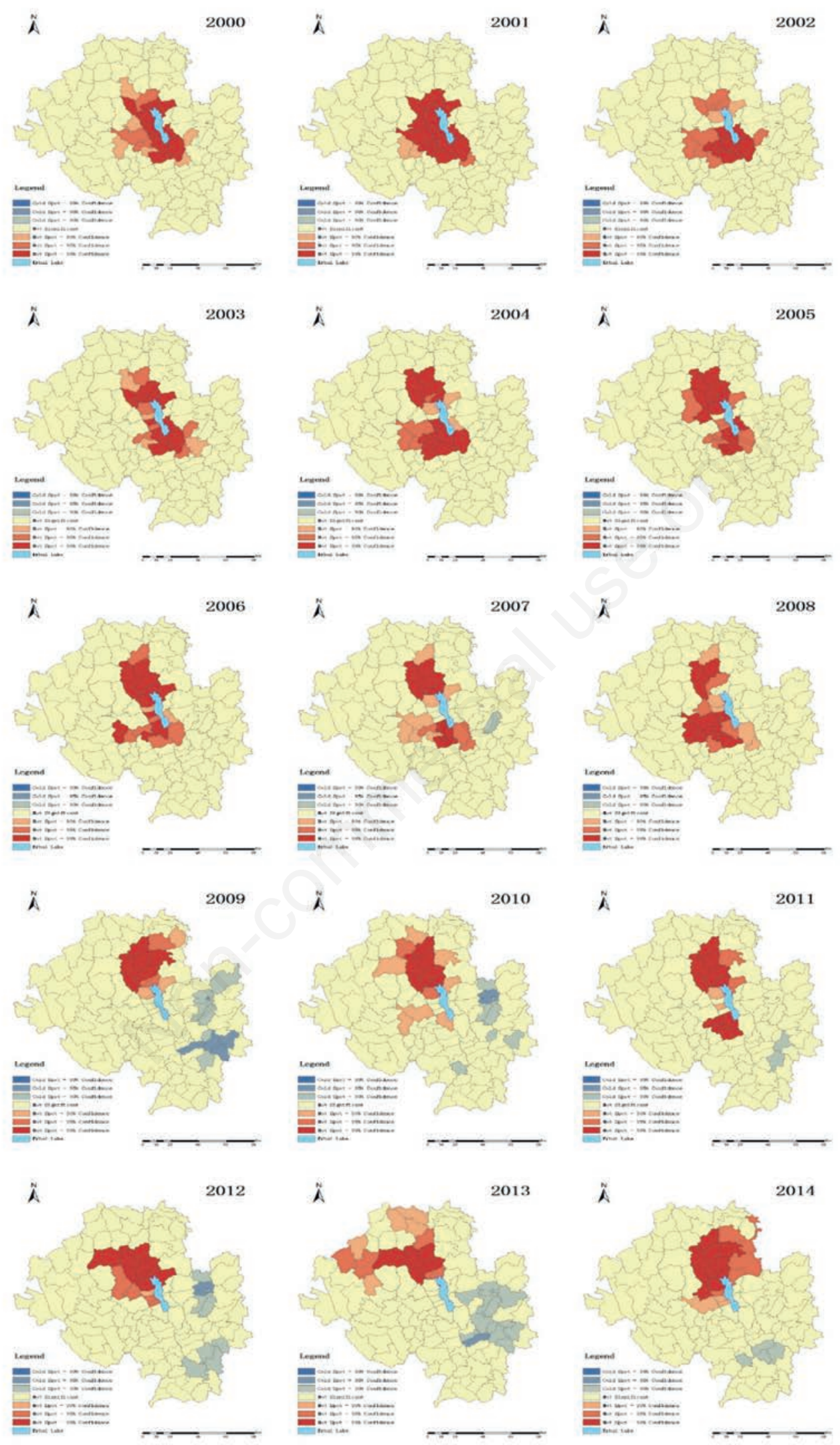

Figure 6. Hotspot analysis. Results based on SaTScan and ArcGIS. 
Dali Prefecture received 38,494,400 visits in 2016, including 934,400 international tourists leading to the possibility of cysticercosis spreading globally (Braae et al., 2017; RodriguezMorales et al., 2017). Influence from local customs and food culture could lead to ingestion of infected raw meats and vegetables by the tourists increases the risk for direct or indirect cysticercosis transmission. Hence, it is necessary to work assiduously to curtail cysticercosis transmission in key areas.

We used the combination of spatial autocorrelation and cluster analysis to define the distribution of cysticercosis in Dali Prefecture. However, cysticercosis incidences considered in this study are limited to cases documented in the clinical in-patient department at the Dali Prefectural Institute of Research and Control on Schistosomiasis. Also, the number of patients with severe cysticercosis may erroneously appear to be reduced, while the real issue could be limited availability of beds and treatment regimes that can take a long time.

Although data from the National Ministry of Health sources are reliable and persuasive, cysticercosis case reporting is not yet standardized in China, which means that only a few areas are delineated as endemic. China is, however, pursuing incorporating cysticercosis into the disease reporting system database, which will facilitate quality data management and also improve future cysticercosis research.

\section{Conclusions}

This study explored the distribution characteristics of cysticercosis cases in Dali Prefecture using spatial autocorrelation and cluster analysis. We found that hotspots were mainly concentrated in the towns in Dali, Wuyuan and Yangbi counties. The continued cysticercosis occurrences in these areas may be attributed to poor environmental factors and unhealthy lifestyles among population. It is, therefore, necessary to carry out cysticercosis prevention and control measures, leveraging multisectoral platforms such as medical promotion, food safety, animal quarantine, environmental protection and health education in areas with high disease prevalence to reduce cysticercosis burden.

\section{References}

Braae UC, Devleesschauwer B, Sithole F, Wang Z, Willingham AL, 2017. Mapping occurrence of Taenia solium taeniosis/cysticercosis and areas at risk of porcine cysticercosis in Central America and the Caribbean basin. Parasit Vectors 10:424.

Chen F, Chen SR, Fang W, Li YL, Luo TP, 2007. [Retrospective analysis on inpatient cases of cysticercosis in 1991-2001 in Dali Prefecture.] J Pathogen Biol 5:380-382. Available from:http://www.cjpb.org.cn/artshow.asp?id=2384 [Article in Chinese].

Coordinating Office of the National Survey on the Important Human Parasitic Diseases, 2005. [A National Survey on Current Status of the Important Parasitic Diseases in Human Population.] Chin J Parasitol Parasit Dis 23:332-40. [Article in Chinese].

Fang W, Bao HE, Huang J, Lang SY, 2009. [The current situation of taeniasis/cysticercosis in Yajiang Area of Sichuan Province and Ming Area of Gansu Province.] J Pathogen Biol 2:121-3. Available from:http://http://www.cjpb.org. cn/artshow.asp?id=1112 [Article in Chinese].

Flisser A, Correa D, 2010. Neurocysticercosis may no longer be a public health problem in Mexico. PLoS Negl Trop Dis 4:e831.

Garcia HH, Del Brutto OH, 2003. Imaging findings in neurocysticercosis. Acta Trop 87:71-8.

Garcia HH, Gonzalez AE, Evans CA, Gilman RH, 2003. Taenia solium cysticercosis. Lancet 362:547-56.

Gripper LB, Welburn SC, 2017. The causal relationship between neurocysticercosis infection and the development of epilepsy - a systematic review. Infect Dis Poverty 6:31.

Gong MQ, Li LX, Zhao ZP, 1997. [Epidemic situation, harm and control countermeasures of taeniasis and cysticercosis in China.] Bull Dis Contr Prevent (China) 1:109-111. Available from: https://dfbt.cbpt.cnki.net/WKB/ WebPublication/ wkTextContent.aspx? colType $=4 \& y \mathrm{t}=1997 \quad \& \mathrm{tp}=\mathrm{gklb}$ [Article in Chinese].

Kulldorff M, 2018. Information Management Services, Inc. SaTScanTM v9.6: Software for the Spatial and Space-Time Scan Statistics. Available from: http://www.satscan.org/

Mahanty S, Garcia HH, 2010. Cysticercosis and neurocysticercosis as pathogens affecting the nervous system. Prog Neurobiol 91:172-84.

Mitchell A, 2005. The ESRI Guide to GIS Analysis. ESRI Press, Redlands, pp. 98-100Available from: https://esripress.esri. com/display/index.cfm?fuseaction $=$ display \&websiteID $=86 \&$ moduleID $=0$

National Bureau of Statistics, People's Republic of China, 2016. [Division codes and urban-rural division codes for statistics in 2016. National Bureau of Statistics, People's Republic of China.] Available from: http://www.stats.gov.cn/tjsj/ tjbz/tjyqhdmhcxhfdm/2016/index.html [Website in Chinese].

Raina SK, Razdan S, Pandita KK, Sharma R, Gupta VP, Razdan S, 2012. Active epilepsy as indicator of neurocysticercosis in rural northwest India. Epilepsy Res Treat 2012:802747.

Rodriguez-Morales AJ, Yepes-Echeverri MC, Acevedo-Mendoza WF, Marin-Rincon HA, Culquichicon C, et al., 2017. Mapping the residual incidence of taeniasis and cysticercosis in Colombia, 2009-2013, using geographical information systems: Implications for public health and travel medicine. Travel Med Infect Dis 22:51-7.

Sheng HF, Yang P, 2011. [Neglected tropical diseases: global impacts and control strategies.] People's Medical Publishing House, Beijing, pp. 86-90. [Book in Chinese]

Tang LH, Xu LQ, Chen YD, 2012. [Control and research on parasitic diseases in China.] Beijing Science and Technology Press, Beijing, pp. 540-565. [Book in Chinese]

Trevisan C, Devleesschauwer B, Schmidt V, Winkler AS, Harrison W, Johansen MV, 2017. The societal cost of Taenia solium cysticercosis in Tanzania. Acta Trop 165:141-54.

Wu W, Jia F, Wang W, Huang Y, Huang Y, 2013. Antiparasitic treatment of cerebral cysticercosis: lessons and experiences from China. Parasitol Res 112:2879-90.

$\mathrm{Xu}$ LQ, 2016. [Illustrated parasitology and parasitoses.] Beijing Science and Technology Press, Beijing, pp. 794-826. [Book in Chinese]

Yu HB, Tang WR, Huang YY, Iang BZE, 2015. [Epidemic Status of Taeniasis and Cysticercosis in Danba County, Sichuan 
Province.] Parasitoses Infect Dis 2:80-2. Available from: http://www.cnki.com.cn/Article/CJFDTotal-SJCB20150 2007.htm [Article in Chinese].

Yu SH, Xu LZ, Jiang ZX, Xu SH, Han JJ, et al., 1994. Report on the first national survey of human parasite distribution. Chin J Parasitol Parasit Dis 12:241-7.

Zammarchi L, Strohmeyer M, Bartalesi F, Bruno E, Munoz J, et al., 2013. Epidemiology and management of cysticercosis and Taenia solium taeniasis in Europe, systematic review 1990-2011. PLoS One 8:e69537.

Zang XZ, Li HZ, Liu HK, Liu YH, Zhou CH, et al., 2019. [Epidemiological analysis of new cases of cysticercosis in
Dali Prefecture, Yunnan Province from 2014 to 2017.] Chin J Schisto Control 31:143-7. [Article in Chinese].

Zhang LL, Yang HM, Yang YL, 1991. [Investigation of parasitic infection in populations in Pumi inhabited areas of Yunnan province.] Chin J Parasitol Parasit Dis S1:153Available from: http://www.jsczz.cn/CN/Y1991/V9/IS1/153 [Article in Chinese].

Zhang MG, 2017. Yunnan yearbook: Yunnan yearbook association, Kunming, p 628. Available from: http://stats.yn. gov.cn/tjsj/tjnj/201810/t20181030_809120.html [Website in Chinese] 